Historic, Archive Document

Do not assume content reflects current scientific knowledge, policies, or practices. 

For Nurserymen Only.

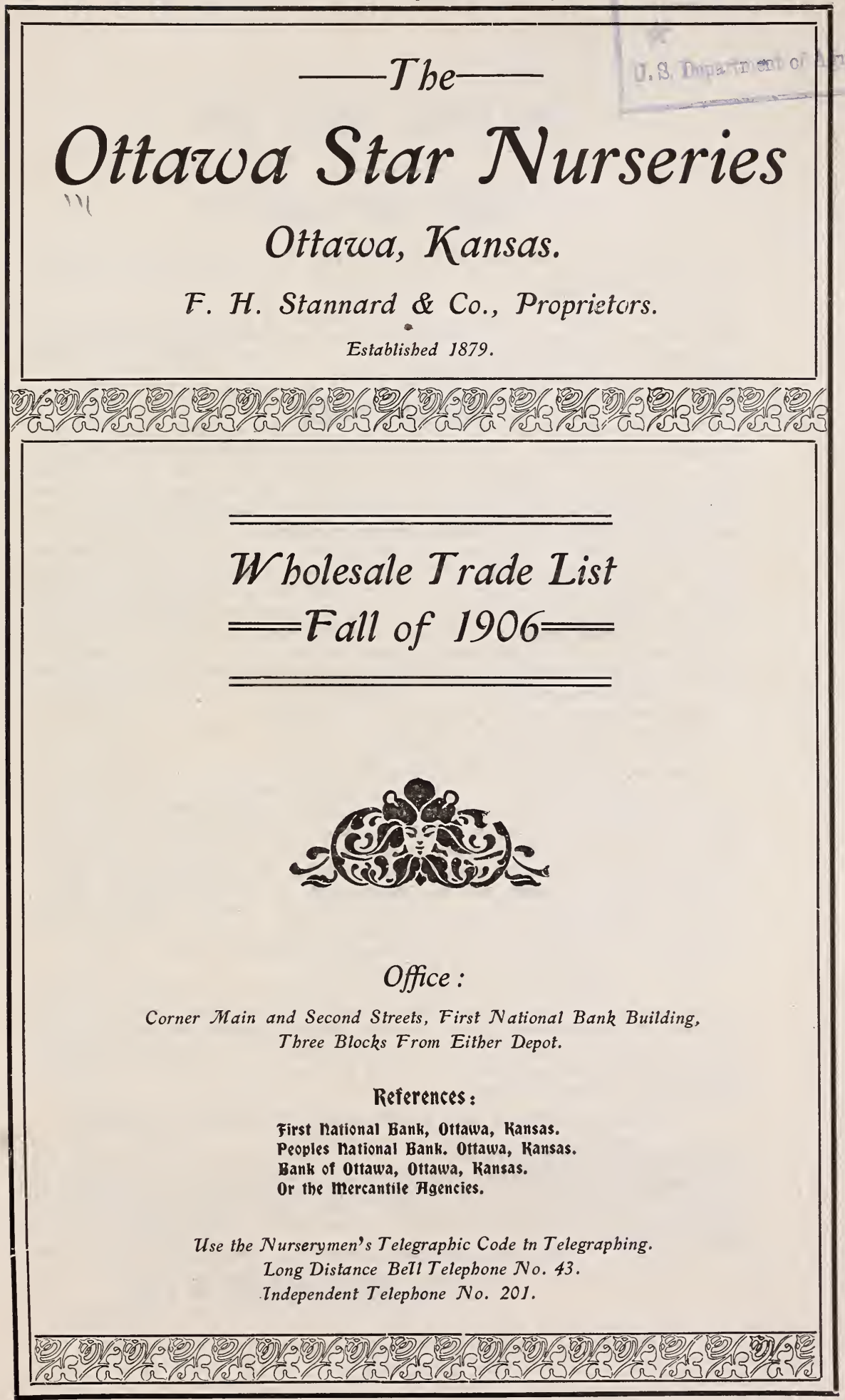




\section{WHOLESALE TRADE LIST.}

\section{FALL OF 1906.}

We present herewith our semi-annual trade list for the fall of 1906, and solicit your patronage. This is our twenty-eighth year in the nursery business. Visitors always welcome.

We have excellent packing facilities and are on the Atchison, Topeka and Santa Fe and Missouri Pacific Railways, with a switch through our packing house, about fifty-five miles southwest of Kansas City, two express companies, the Wells Fargo \& Co., and the Pacific Express Company.

\section{Terms of Sale.}

Our shipping season begins October first and continues until April fifteenth. Please furnish explicit shipping directions, mentioning the route, and whether you wish shipment by express or freight. When no directions are given, we use our judgment, but in no case do we assume any responsibility as to safe arrival and condition of stock after a proper shipment.

We grade carefully; in some cases by caliper, others by height. When by both, the caliper must govern. The lower number is included and the higher number is excluded. For instance, in a grade of four to five feet, the four feet trees are included, but the five feet trees go into the next grade higher. When desired, will send samples to prospective customers.

We pack all stock in the most thorough manner, for which a moderate charge-sufficient to cover the cost-will be made. Boxes packed and paper-lined will be charged as follows:

30 inches square, 8 to 10 feet long, at $\$ 3.00$ each.

24 inches square at $\$ 2.00$ to $\$ 2.50$ each.

18 inches square at $\$ 1.50$ to $\$ 1.75$ each.

Bales at from 25 cents to $\$ 1.00$ each.

Cash or satisfactory security before shipment. Remit by Kansas City or eastern exchange, or express money order. Orders to be sent C. O. D. must be accompanied by at least one-fourth the amount of the order. To promptpaying customers, the usual thirty to sixty days will be allowed from the date of shipment, but where the time is not especially agreed upon, thirty days is to be understood. Interest will be charged upon all overdue accounts. Should prices be changed, either higher or lower, during the season, the prices ruling at the time the order is made are expected to stand. When the order amounts to $\$ 50.00$ or over, the articles will be figured at thousand rates.

Prices are subject to change without notice.

While we exercise the greatest diligence and care to have all our trees and plants true to label, and hold ourselves in readiness, on proper proof, to replace all trees that may prove untrue to labels, free of charge or refund the amount paid, it is mutually understood and agreed between the purchaser and ourselves that our guarantee of genuineness shall in no case make us liable for any sum greater than originally received.

\section{A Health Certificate Will Be Furnished With Each Shipment.} UNIVERSITY OF KANSAS, \} NO. 76

CBRTIFICATE OF NURSEUY INSPECTION, 作 nurserv stock and premises of F. H. STANNARD \& Co., situated at OTTAWA, KANSAS, have been duly inspected and there has been found thereon no indications of the presence of the San Jose scale or other dangerous insect or plant disease. This certificate is invalid after June 1st, 1907.

S.J. BUNTER.

Head of Department of Entomology, State Inspector of Nurseries.

\section{F. H. STANNARD \& CO.}




\section{FRUIT DEPARTMENT.}

\begin{tabular}{|c|c|c|c|}
\hline \multirow[b]{2}{*}{ Two Years, Smooth. } & APPLES. & & \\
\hline & Code Word. & Per 100. & Per 1000. \\
\hline 5 to $6 \mathrm{ft} ., 3 / 4$ in. and up & .....Gabble & $\$ 6.50$ & $\$ 55.00$ \\
\hline 4 to $6 \mathrm{ft}$., $5 / 8$ in. to $3 / 4$ in. & ......Gad & 5.00 & 45.00 \\
\hline 1 to $5 \mathrm{ft} ., 1 / 2$ to $5 / 8$ in..... & ...... Gaff & 4.00 & 35.00 \\
\hline 3 to $4 \mathrm{ft}$, mostly branch & .....Gage & 3.00 & 25.00 \\
\hline
\end{tabular}

Varieties marked with a star will be furnished in such proportion in assortment as our stock will permit, and if supplied alone will be charged for at one cent per tree additional for the two upper grades.

\begin{tabular}{|c|c|c|}
\hline *Arkansas Black & Longfield & Rawles' Genet \\
\hline Autumn Strawberry & Lowell & *Rhode Island Greening \\
\hline *Alexander & Loy & Salome \\
\hline *Bailey Sweet. & Limber Twig & Scott's Winter \\
\hline Baldwin & Maiden Blush & Smith's Cider \\
\hline Bellflower & Mann & *Spitzenberg \\
\hline Ben Davis & Martha (Crab) & Stark \\
\hline Benoni & Nammoth Black Twig & Springdale \\
\hline Clayton & IMcAfee's Nonesuch & Summer Queen \\
\hline Cooper's Early White & $*$ McIntosh Red & *Sweet Bough \\
\hline Chenango Strawberry & McMahon's White & *Talman Sweet \\
\hline Dominie & Minkler & *Tetofsky \\
\hline$*$ Dutchess of Oldenburs & Missouri Pippin & Twenty-ounce Pippin \\
\hline *Early Harvest & Northern Spy & Transcendent (Crab) \\
\hline Fall Pippin & Northwestern Greening & Utter's Red \\
\hline Fameuse & Penn. Red Streak & Vandevere Pippin \\
\hline Flora Belle & Pewaukee & Wagoner \\
\hline Gano & Plum Cider & Walbridge \\
\hline General Grant (Crab) & *Rambo & *Wealthy \\
\hline Grimes' Golden Pippin & $*$ Red Astrachan & Willow Twig \\
\hline Golden Sweet & $*$ Red June & White Arctic (Crab) \\
\hline Haas & Red Romanite & White Winter Pearmain \\
\hline Fluntsman's Favorite & Red Winter Pearmain & *Whitney No. 20 (Crab) \\
\hline *Hibernal & Red Beitigheimer & Winesap \\
\hline Hyslop (Crab) & Red Siberian (Crab) & *Wolf River \\
\hline Iowa Blush & Rome Beauty & York Imperial \\
\hline Jonathan & Roman Stem & *Yellow Transparent \\
\hline
\end{tabular}

These Apples are very fine. We have never grown a more handsome lot. 


\section{PEARS.}

Two Years-Standard.

Code Word.

Per 100. Per 1000.

5 to $7 \mathrm{ft}$., No. $1,3 / 4$ in. and up..... Gaiety

$\$ 14.00$

$\$ 120.00$

5 to $6 \mathrm{ft}$., No. $1,5 / 8$ to $3 / 4$ in..... Gainish

12.00

100.00

4 to $5 \mathrm{ft}$., No. $1,1 / 2$ to $5 / 8$ in.......Galaxy

9.00

80.00

$31 / 2$ to $5 \mathrm{ft}$., mostly branched......Galena

Bartlett

Beurre de Anjou

Belle Lucrative

Clapp's Favorite

Flemish Beauty

Garber

Howell

Dwarf.

4 to $5 \mathrm{ft} ., 3 / 4$ in. and up, No. 1.... Gallant

4 to $5 \mathrm{ft}$., $5 / 8$ to $3 / 4$ in., No. 1..... Galleon

3 to $4 \mathrm{ft}$., $1 / 2$ to $5 / 8$ in., No. $1 \ldots$... Gallery

Partlett

Beurre de Anjou

Buffum

Garber

Howell

Clapp's Favorite

Keiffer

Doyenne Boussock

Koonce

Duchess

Flemish Beauty

Lawson

Lincoln

Louise Bon

\section{PEACH.}

One Year.

Code Word.

No. 1,5 to $6 \mathrm{ft} ., 5 / 8$ in. and up. . Galvanism

No. 1,4 to 5 ft., $9-16$ to $5 / 8$ in.... Gambit

3 to $4 \mathrm{ft}$., $7-16$ to $9-16$ in .......Gambage

3 to $4 \mathrm{ft}$, under $7-16$ in.. .. . . Gambrel

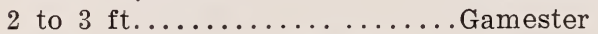

Amsden

Emma

Alexander

Arkansas Traveler

Bloodleaf

Belle of Georgia

Captain Ede

Carman

Champion

Chair's Choice

Crawford's Early

Crawford's Late

Coolidge's Favorite

Crosby

Elberta

Early Rivers

Early York
Family Favorite

Foster

Fitzgerald

Fox's Seedling

Greensboro

Gold Drop

Hale's Early

Heath Cling

Heath Free

Hill's Chili

Horton's Rivers

Kalamazoo

Lemon Free

Lemon Cling

Lewis Seedling
7.00

60.00

Rutter

Seckel

Sheldon

Tyson

Vermont Beauty

Vicar of Wakefield

Wilder

$\begin{array}{rr}\$ 9.00 & \$ 85.00 \\ 8.00 & 70.00 \\ 6.50 & 55.00\end{array}$

Mit. Vernon

Rutter

Seckel

Sheldon

Tyson

Wilder

Per 100. Per 1000.

$\$ 6.00$

5.00

$\$ 55.00$

4.50

45.00

3.50

2.50

Mit. Rose

Old Mixon Free

Piquets' Late

Reeves' Favorite.

Salway

Stephens' Rareripe

Stump the World

Smock

Sneed

Susquehanna

Triumph

Wonderful

Waterloo

Wager

Wheatland

Wright's Seedling 


\section{CHERRIES ON MAHALEB.}

Two Years.

Code Word.

Per 100. Per 1000.

No. $1,4 \frac{1}{2}$ to $6 \mathrm{ft} ., 3 / 4$ in. and up....Gallic

$\$ 20.00$

$\$ 185.00$

No. 1,4 to $5 \mathrm{ft}$., $5 / 8$ to $3 / 4$ in....... Galloon

17.00

160.00

3 to $4 \mathrm{ft}$, $1 / 2$ to $5 / 8$ in............ Gallows

11.00

100.00

3 to $4 \mathrm{ft}$., $3 / 8$ to $1 / 2$ in.......... Galache

8.00

70.00

$\begin{array}{lll}\text { Black Tartarian } & \text { Governor Wood } & \text { May Duke } \\ \text { Dyehouse } & \text { Late Duke } & \text { Olivet } \\ \text { Early Richmond } & \text { Lieb } & \text { Ostheimer } \\ \text { Empress Eugenie } & \text { Louis Phillip } & \text { Wragg } \\ \text { Fnglish Morello } & \text { Montmorency } & \text { Yellow Spanish }\end{array}$

Sweet Cherries 2c additional in two upper grades.

\section{ALMOND ON PEACH.}

One Year.

Code Word.

Per 100. Per 1000.

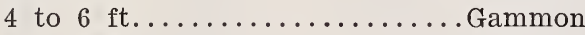

4 to $5 \mathrm{ft} . \ldots \ldots \ldots \ldots \ldots \ldots$. Ganglion

$\$ 12.00$

$\$ 120.00$

9.00

80.00

\section{NATIVE PLUM ON PLUM.}

One and Two Years.

Code Word.

Per 100. Per 1000.

No. 1,5 to $7 \mathrm{ft.}, 3 / 4$ in. and up... Gangrene

5 to $6 \mathrm{ft}$., $5 / 8$ to $3 / 4$ in.......... Gangway

4 to $5 \mathrm{ft}$., $1 / 2$ to $5 / 8$ in......... Gargarize

3 to $5 \mathrm{ft}$., mostly branched.... . Garland

$\$ 16.00$

14.00

$\$ 150.00$

12.00

130.00

100.00

8.00

70.00

\section{EUROPEAN AND JAPAN PLUM ON PLUM.}

Two Years.

Cođe Word.

Per 100.

Per 1000

No. 1,5 to $6 \mathrm{ft} ., 3 / 4$ in. and up.....Gamer

No. 1,5 to $6 \mathrm{ft}$, $5 / 8$ to $3 / 4$ in.... Gamiture

$\$ 13.00$

11.00

$\$ 120.00$

No. 1,4 to $5 \mathrm{ft}$., $1 / 2$ to $5 / 8$ in..... Garrison

9.00

100.00

No. 1,3 to $4 \mathrm{ft}$., mostly branched..Garter

8.00

80.00

70.00

\section{JAPAN AND NATIVE PLUM ON PEACH.}

One and Two Years.

Code Word. Per 100 Per 1000

No. 1,5 to $6 \mathrm{ft} ., 3 / 4$ in. and up.... Gaskins

$\$ 12.00$

10.00

No. 1,4 to $5 \mathrm{ft}$., $5 / 8$ to $3 / 4$ in.......Gastric

$\$ 100.00$

No. 1,4 to $5 \mathrm{ft}$., $1 / 2$ to $5 / 8$ in....... Gaudy

7.00

80.00

65.00

$31 / 2$ to $5 \mathrm{ft}$., mostly branched...... Guage

\section{Leading Varieties of Native Plums.}

Ine Soto

Forest Garden

Golden Beauty

Hawkeye

\section{Mariana}

Milton

Pottawatomie

Robinson
Wolf

Weaver

Wild Goose 


\begin{tabular}{lcc} 
& Leading Varieties of European Plums. \\
Bradshaw & General Hand & Pond Seedling \\
Blue Damson & Genii & Reine Claude \\
Empire & Grand Duke & Saratoga \\
F. Damson & Imperial Gage & Smith Orleans \\
Fellenberg & Lombard & Shipper's Pride \\
Coe's Golden Drop & Moore's Arctic & Shropshire Damson \\
German Prune & Niagara & Yellow Egg \\
\multicolumn{1}{c}{ Burbank } & Leading Varieties Japan Plums. & \\
Botan & Prunus Pissardii & Willard \\
Hale & Red June & Wickson \\
Ogon & Satsuma & Simoni
\end{tabular}

APRICOT ON PEACH.

One and Two Years.

Code Word. Per 100 Per 1000

\begin{tabular}{|c|c|c|}
\hline No. 1,5 to $6 \mathrm{ft} ., 3 / 4$ in, and up.... Gauntlet & $\$ 12.00$ & $\$ 100.00$ \\
\hline No. 1,4 to $6 \mathrm{ft} ., 5 / 8$ to $3 / 4$ in....... Gavel & 12.00 & 100.00 \\
\hline No. 1,3 to 5 ft., $1 / 2$ to $5 / 8$ in...... Gazette & 8.00 & 80.00 \\
\hline 3 to $4 \mathrm{ft}$., mostly branched .........Gear & 7.00 & 60.00 \\
\hline $\begin{array}{l}\text { Early Golden } \\
\text { Gibb }\end{array}$ & $\begin{array}{l}\text { Mo } \\
\text { Ro }\end{array}$ & \\
\hline
\end{tabular}

Superb

NECTARINE.

One Year. Code Word. Per 100. Per 1000.

No. $1,41 / 2$ to $6 \mathrm{ft} \ldots \ldots \ldots \ldots \ldots$.......................... $\$ 15.00$

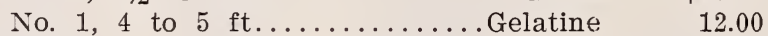

IBoston

Two Years. $\quad$ Code Word. Per 100. Per 1000.

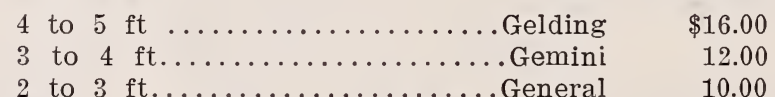

2 to $3 \mathrm{ft} . \ldots \ldots \ldots \ldots \ldots \ldots$................... 10.00

Champion Mo. Mammoth Orange

Meech's

GRAPES.

Code Word. Per 100. Per 1000.

Agawam, 1 yr., No. 1............Generic

$\$ 2.50$

$\$ 20.00$

Agawam, 2 yrs., No. 1............ Genet

25.00

Brighton, 1 year., No. 1..........Genius

2.50

20.00

Brighton, 2 yrs., No. 1........... Genteel

Concord, 1 yr., No. 1...........Gentian

Concord, 2 yrs., No. 1 .........Genuine

Campbell's Early, 1 yr., No. 1....Geogony

Champion, 1 yr., No. 1.......Geography

Champion, 2 yrs., No. 1.........Georgic

Catawba, 1 yr., No. 1............. Gerin

Catawba, 2 yrs., No. 1...........German

Clinton, 1 yr., No. 1............Gerund 
Delaware, 1 yr., No $1 \ldots \ldots \ldots \ldots$ Gewgaw

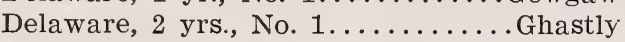

Elvira, 1 yr, No. 1...........Gherkin

Elvira, 2 yrs., No. $1 \ldots \ldots \ldots \ldots \ldots$ Ghost

Empire State, 1 yr., No. 1........Ghoul

Empire State, 2 yrs., No. 1......... Giant

Eaton, 1 yr., No. 1.............. Gibbet

4.00

Eaton, 2 yrs., No. 1.............Giddy

Hartford, 1 yr., No. 1............ Giggle

Hartford, 2 yrs., No. 1.............Gild

Ives' Seedling, 1 yr., No. 1......Gimcrack

Ives' Seedling, 2 yrs. No. 1 .........Gimlet

Lady, 1 yr., No. $1 \ldots \ldots \ldots \ldots \ldots$. Ginger

3.00

Lady, 2 yrs. No. 1.............Ginseng

4.00

Lindley, 1 yr., No. $1 \ldots \ldots \ldots \ldots$....... Giraffe

Moore's Early, 1 yr., No. 1.....Girandole

Moore's Early, 2 yrs., No. 1.........Girder

Moore's Diamond, 1 yr., No. 1.......Girth

Moore's Diamond, 2 yrs., No. 1..... Gizzard

Martha, 1 yr., No. $1 \ldots \ldots \ldots \ldots$...............

Martha, 2 yrs., No. 1............Gladden

Niagara, 1 yr., No. $1 . \ldots \ldots$..... Gladiator

Niagara, 2 yrs., No. 1..........Gladsome

Pocklington, 1 yr., No. $1 \ldots \ldots$....Glamour

Pocklington, 2 yrs., No. 1........Glance

Salem, 1 yr., No. 1............Glanders

Salem, 2 yrs., No. 1...........Glandular

Vergennes, 1 yr., No. 1.........Glaring

Vergennes, 2 yrs., No. 1.......Glaucous

Worden, 1 yr., No. $1 \ldots \ldots \ldots \ldots \ldots$. . Glave

Worden, 2 yrs., No. $1 \ldots \ldots \ldots$....Glazier

Wilder, 1 yr., No. $1 \ldots \ldots \ldots \ldots \ldots$. Gleam

3.50

4.00

3.00

3.50

30.00

Wyoming Red, 1 yr., No. $1 \ldots \ldots \ldots$. Gleeful

Wyoming Red, 2 yrs., No. 1........Glibly

35.03

No. 2 Vines of the above assortment will be supplied at 70 per cent of the above prices. They are of good quality. Samples will be sent by mail on application.

\section{CURRANTS.}

Code Word. Per 100. Per 1000

Red Dutch, Victoria, 2 yrs., No. 1..Glimmer

Red Dutch, Victoria, 1 yr., No. 1....Glimpse

$\$ 3.50$

2.50

3.50

2.50

3.50

3.00

4.00

3.00

4.00
$\$ 30.00$

20.00

30.00

20.00

30.09

20.00

35.00

25.00

35.00

North Star, 1 yr., No. 1..........Glorify 
GOOSEBERRIES.

Code Word. Per 100. Per 1000.

Downing, 2 yrs., No. 1.............. Gloss

$\$ 6.00$

Houghton, 2 yrs., No. 1..........Glossary $\$ \$ 5.00$

Smith's Improved, 2 yrs., No. 1....Glottis $\quad 6.00$

Champion, 2 yrs., No. 1...........Glout $\quad 7.00$

Industry, 2 yrs., No. $1 \ldots \ldots \ldots \ldots$. Glow $\quad 10.00$

Chautauqua, 1 yr., No. 1.......... Glue 12.00

Josselyn, 1 yr., No. 1............ Gluten $\quad 7.00$

BLACKBERRIES.

Code Word. Per 100. Per 1000.

Larly Harvest, root-cutting plants..Glyptic

Snyder, root-cutting plants..........Gnarl

$\$ .75$

1.00

$\$ 7.00$

Firie, root-cutting plants............ Gnash

1.20

10.00

Kittatinny, root-cutting plants........ Gnat

1.00

10.00

Stone's Hardy, root-cutting plants..Gneiss

Rathbun, root-cutting plants........ Gnome

1.00

10.00

Ancient Britain, root-cutting plants.Gnomon

2.50

10.00

1.50

20.00

RASPBERRIES.

Code Word. Per 100. Per 1000.

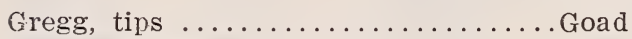

Souhegan, tips $\ldots \ldots \ldots \ldots \ldots \ldots$ Gobbler

Ohio, tips. ......................

Ilopkins, tips $\ldots \ldots \ldots \ldots \ldots \ldots$ Go-cart

Mammoth Cluster, tips...........Goddess

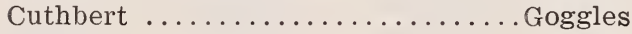

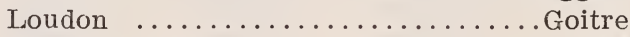

Kansas, tips .........................

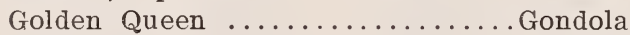

Cardinal ...............................

$\begin{array}{rr}\$ .75 & \$ 6.00 \\ .75 & 6.00 \\ .75 & 6.00 \\ .75 & 6.00 \\ .75 & 6.00 \\ .90 & 6.00 \\ 1.25 & 10.00 \\ .75 & 5.00 \\ 1.00 & 10.00 \\ 2.00 & 15.00\end{array}$

STRAWBERRIES.

Code Word-Gorge. Per 100, 35c. Per 1000, $\$ 3.00$.

Leading Varieties.

$\begin{array}{llll}\text { Bederwood } & \text { Captain Jack } & \text { Jesse } & \text { Rough Rider } \\ \text { Brandywine } & \text { Charles Downing } & \text { Michel's Early } & \text { Splendid } \\ \text { Bubach } & \text { Gandy } & \text { Nich Ohmer } & \text { Senator Dunlap } \\ \text { Crescent } & \text { Greenville } & \text { New York } & \text { Sample } \\ \text { Cumberland } & \text { Haverland } & \text { Kobinson } & \text { Warfield }\end{array}$

MISCELLANEOUS.

Code Word. Per 100. Per 1000.

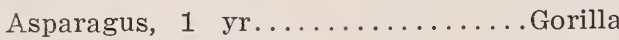

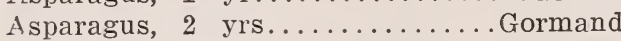

$\$ .40$

.50

$\$ 4.00$

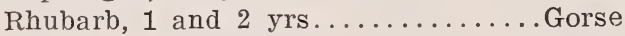

2.50

5.00

Dwarf Juneberries ............Goshawk

4.00

20.00

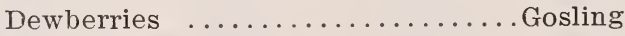

1.00

35.00

Strawberry-Raspberry ...........Gospel

2.00

10.00

Horse Radish ...............Gossamer

1.50

15.00

Buffalo Berries ...............Gossip

15.00 


\section{PERSIMMONS.}

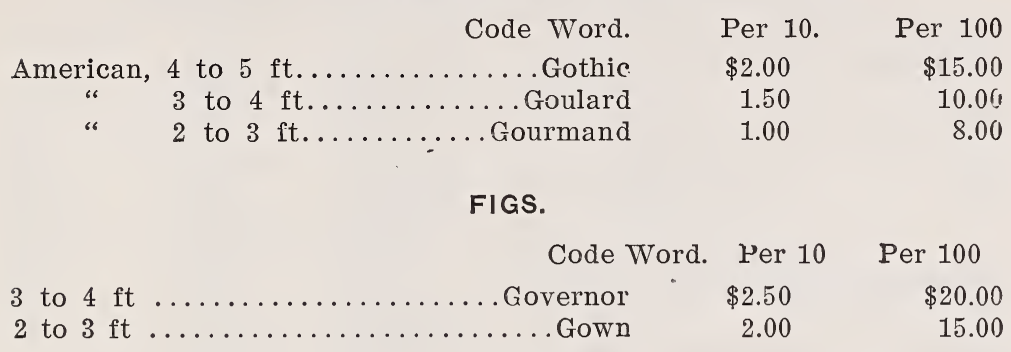

\section{DECIDUOUS ORNAMENTAL TREES.}

We have a good stock of nursery grown shade and ornamental trees. They have been transplanted and are suitable for street and park planting.

Code Word. Per 10 Per 100

Ash, Am. White, trans., 4 to $5 \mathrm{ft} . . . . .$. Grail

$\begin{array}{rr}.50 & 4.00 \\ .75 & 6.00 \\ 1.00 & 8.50 \\ 1.50 & 14.00 \\ 3.00 & 25.00 \\ .50 & 6.00 \\ .75 & 7.00 \\ 1.00 & 10.00 \\ 1.50 & 15.00 \\ 1.00 & \\ 1.20 & \\ 1.50 & \\ 1.80 & \\ 3.00 & \\ 1.00 & 5.00 \\ 1.25 & 8.00 \\ 1.25 & 10.00 \\ 1.50 & 12.50 \\ 2.00 & 15.00 \\ 2.50 & 20.00 \\ .50 & 4.00 \\ .75 & 6.00 \\ 1.00 & 8.00 \\ 1.50 & 12.50 \\ 2.00 & 18.00 \\ 2.50 & 20.00 \\ 3.50 & 30.00 \\ 4.00 & 35.00 \\ 3.50 & 30.00 \\ 3.00 & 25.09 \\ 1.25 & 8.00 \\ 1.50 & 10.00 \\ 1.75 & 12.00 \\ 2.00 & 15.00 \\ & \end{array}$

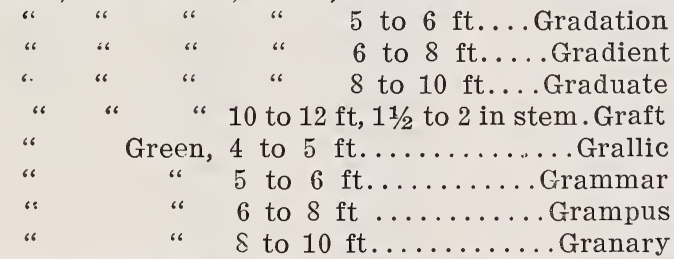

Eirch, Europ'n White, trans. 3 to $4 \mathrm{ft}$... Grandee

Box Elder, trans., 4 to $5 \mathrm{ft} . . . \ldots \ldots$..... Grape

" " " " " " $\quad$ " $\quad 4$ to $5 \mathrm{ft}$. 6 to $6 \mathrm{ft}$....Grandsire

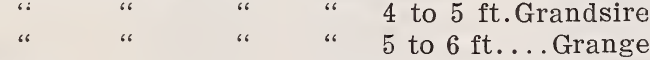

6.00

8.50

14.00

6.00

7.00

10.00

15.00

" " " " " 6 " 6 to $8 \mathrm{ft}$. . Granite

" " " " 5 to $6 \mathrm{ft} . \ldots . \ldots . .$. Graphic

" " " 6 to $8 \mathrm{ft} . . . . . . .$. Grapnel

" " " " 8 to $10 \mathrm{ft} . . . . .$. . Grapple

“ " " 10 to $12 \mathrm{ft} 1 \frac{1 / 2}{2}$ to 2 in stem Grasp

" " " 2 to $2 \frac{1}{2}$ in stem....... Gratify

Catalpa Speciosa, 3 to $4 \mathrm{ft} . . . . .$. Gratitude

4 to 5 ft..........Gratuity

5 to $6 \mathrm{ft} \ldots \ldots \ldots$........ Grave

6 to $8 \mathrm{ft} . . . \ldots$. Gravitate

1 to $1 \frac{1 / 2}{2}$ in 8 to $10 \mathrm{ft}$.... Gray

$11 / 2$ to 2 in 10 to $12 \mathrm{ft}$. Grease

2 to $2 \frac{1}{2}$ in 12 to $15 \mathrm{ft}$. Grecian

Catalpa Bungei, fine, 8 to $10 \mathrm{ft} . . . \ldots$. . Greek

" 6 to $8 \mathrm{ft} . . . \ldots$. Greeting

" 5 to $6 \mathrm{ft} . . .$. Gregarious

Chestnut, Sweet, trans., 3 to $4 \mathrm{ft} . . .$. Grenada

" " " 4 to $5 \mathrm{ft} . .$. Grenadine

5 to $6 \mathrm{ft}$.... Grewsome

6 to $8 \mathrm{ft}$......Griddle

2.00 


\section{DECIDUOUS ORNAMENTAL TREES. (Continued.)}

Code Word. Per 10 Per 100

Chestnut, Horse, trans., 4 to $5 \mathrm{ft}$... Grievance

2.50

" " " 5 to $6 \mathrm{ft}$......Griffin

Elm, Am., trans., 4 to $5 \mathrm{ft} . \ldots \ldots \ldots \ldots$...........

3.50

.80

1.00

6.00

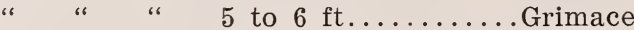

" " " 6 to $8 \mathrm{ft} \ldots \ldots \ldots \ldots$ Grimalkin

1.25

8.00

" " " 8 to $10 \mathrm{ft} . . . . . . . .$. Grime

" " 10 to $12 \mathrm{ft}, 1 \frac{1}{2}$ to 2 in stem..... Grinder

1.50

10.00

3.50

15.00

Hackberry, transplanted, 4 to $5 \mathrm{ft}$........Grip

.75

35.00

" " 5 to $6 \mathrm{ft}$.....Grisette

1.00

6.00

Judas Tree, or Red Bud, 3 to $4 \mathrm{ft}$......Griskin

1.00

1.25

" " " 4 to $5 \mathrm{ft}$ "......Grist

5 to $6 \mathrm{ft} . .$. Grizzle

6 to $8 \mathrm{ft} . . .$. Groan

Locust, Black, 4 to $5 \mathrm{ft} . \ldots \ldots \ldots \ldots$. . . Frocery

1.50

1.75

.50

4.00

.80

6.50

1.00

8.00

1.25

12.00

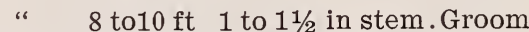

" 10 to $12 \mathrm{ft} 1$ to $1 \frac{1 / 2}{\text { in stem. Grop }}$

Honey, 3 to $4 \mathrm{ft} . . . \ldots . .$. Grotesque

2.50

20.00

.75

1.00

6.00

" 4 to 5 ft............ Grotto

" " 5 to $6 \mathrm{ft} \ldots \ldots \ldots \ldots$ Ground

1.25

8.00

10.00

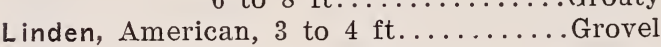

1.50

$\mathbf{1 4 . 9 0}$

" " 4 to $5 \mathrm{ft} \ldots \ldots \ldots \ldots$. Growth

1.00

1.25

1.50

2.50

1.50

European, 4 to $5 \mathrm{ft} . \ldots \ldots$..... Grumble

1.75

5 to $6 \mathrm{ft} . \ldots \ldots$...... Grumous

2.50

Mountain Ash, European, 4 to $5 \mathrm{ft}$......Guard

1.20

8.00

1.50

12.50

1.75

6 to $8 \mathrm{ft}$....Guerdon

1.50

15.00

" oak leaved, 4 to $5 \mathrm{ft}$....Guerilla

12.50

2.00

2.50

Maple, soft or white, trans. 3 to $4 \mathrm{ft}$. . Guidance

soft or white, trans. 6 to $8 \mathrm{ft}$. . Guillotine soft or white, trans. 8 to $10 \mathrm{ft}$

1 to $1 \frac{1}{2}$ in. stem.............. Guitar

soft or white, transplanted

10 to $12 \mathrm{ft} 1 \frac{1 / 2}{2}$ to 2 in, stem... Gulch soft or white, transplanted

12 to $14 \mathrm{ft}, 2$ to $2 \frac{1}{2}$ in stem.... Gullet

Sugar, trans., 4 to $5 \mathrm{ft} . . . \ldots$....Gummy

1.50

Sugar, trans., 5 to $6 \mathrm{ft}$........Gumption

2.00

Sugar, trans., 6 to $8 \mathrm{ft}$........Gunwale

2.50

Norway, trans., 5 to $6 \mathrm{ft}$.......Gurgle

Norway, trans., 6 to $8 \mathrm{ft}$........Gussett

3.50

Norway, trans., 8 to $10 \mathrm{ft}, 1$ to

$1 \frac{1}{2}$ in................... Gutter 


\section{DECIDUOUS ORNAMENTAL TREES. (Continued.)}

Code Word. Per 10 Per 100

Maple, Weir's cut-leaved, trans., 4 to

$$
5 \mathrm{ft} \ldots \ldots \ldots \ldots \ldots \text {. Gutteral } \quad 1.20
$$

10.00

“ Weir's cut-leaved, trans., 5 to

$6 \mathrm{ft}$................ Gymnasium $\quad 1.80$

15.00

"Weir's cut-leaved, trans., 6 to

$8 \mathrm{ft} \ldots \ldots \ldots \ldots \ldots \ldots$ Gypsum.

2.00

18.00

“ Weir's cut-leaved, trans., 8 to

$10 \mathrm{ft}, 1$ to $1 \frac{1}{2}$ in stem....... Gyral

25.00

“ Weir's cut-leaved, trans., 10 to

$12 \mathrm{ft}, 1 \frac{1 / 2}{2}$ to 2 in stem..... Gyration

Mulberry, Russian, 3 to $4 \mathrm{ft} . \ldots \ldots \ldots$...........

3.50

30.00

.50

4.00

Russian, 4 to $5 \mathrm{ft} . . .$. ... Gyroscope

.75

6.00

" Russian, 5 to $6 \mathrm{ft} \ldots \ldots \ldots \ldots$.................

1.00

1.25

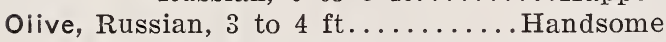

8.00

1.20

“ Russian, 4 to $5 \mathrm{ft} . . . \ldots \ldots$....Haberdine

" Russian, 5 to $6 \mathrm{ft} . \ldots \ldots$.....Habiliment

Poplar, Carolina, 4 to $5 \mathrm{ft} \ldots \ldots \ldots \ldots \ldots$ Habit

Carolina, 5 to $6 \mathrm{ft} \ldots \ldots \ldots \ldots \ldots$

“ Carolina, 6 to $8 \mathrm{ft} \ldots \ldots \ldots \ldots \ldots \ldots \ldots \ldots$

" Carolina, 8 to $10 \mathrm{ft} . \ldots \ldots \ldots$.............

“ Carolina, 10 to $12 \mathrm{ft}, 1$ to $1 \frac{1}{2}$

in. stems ............ Hackney

Carolina, 12 to $14 \mathrm{ft}, 1 \frac{1 / 2}{2}$ to 2

in. stems............... Haddock

1.50

10.00

8.00

10.00

.50

12.00

.60

4.00

1.00

1.25

5.00

8.00

$-$

10.00

1.50

15.00

2.00

1.00

1.20

Lombardy, 6 to $8 \mathrm{ft} \ldots \ldots \ldots$......................

Lombardy, 8 to $10 \mathrm{ft} . . . \ldots \ldots$..... Hail

Lombardy, 10 to $12 \mathrm{ft}, 1$ to

$1 \frac{1}{2}$ in. stems............ Hairiness

1.50

18.00

8.00

10.00

12.00

2.00

20.00

1.00

Balm of Gilead, 5 to $6 \mathrm{ft}$.... Halberd

1.25

8.00

Balm of Gilead, 8 to $10 \mathrm{ft}, 1$ to

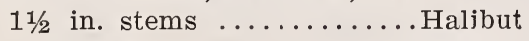

sycamore, 3 to $4 \mathrm{ft} . \ldots \ldots \ldots \ldots \ldots$.............

1.50

10.00

.60

12.00

4 to $5 \mathrm{ft} . \ldots \ldots \ldots \ldots$ Halloween

.75

5.00

1.00

5 to $6 \mathrm{ft} \ldots \ldots \ldots \ldots \ldots \ldots$. Halter

1.50

6.00

8.00

6 to $8 \mathrm{ft} . \ldots \ldots \ldots \ldots \ldots$ Halyard

$11 / 2$ in. stems ..........Hammock

10 to $12 \mathrm{ft} ; 11 / 2$ to

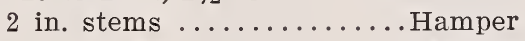

1.80

15.00

3.00

25.00

1.20

10.00

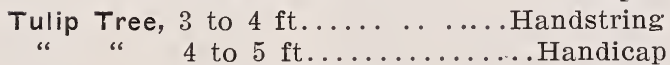

1.50

15.00

2.00

18.00

Willow, Golden, 5 to $6 \mathrm{ft} \ldots \ldots \ldots \ldots$ Happily

.75

1.00

Walnut, Black, 5 to $6 \mathrm{ft} \ldots \ldots \ldots \ldots$ Harangue 


\section{EVERGREENS.}

Code Word. Per 10

Arbor Vitae, American, 2 to $3 \mathrm{ft}$.... Harbinger

$\begin{array}{cc}\text { " } & \text { "6 } \\ \text { " } & \text { " } \\ \text { " } & \text { " }\end{array}$

"6

" 18 to 24 in.... Harbor

Chinese, 2 to $3 \mathrm{ft}$.........Hardly

" $18^{\text {" to }} 24$ in... Hardship

Siberian, 18 to 24 in... Hardware

" 12 to 18 in...... Hare

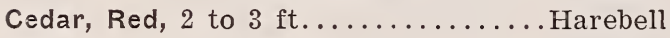

" " 18 to 24 in............... Harelip

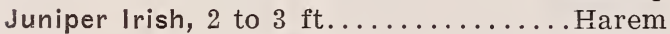

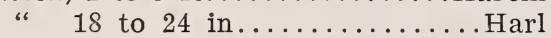

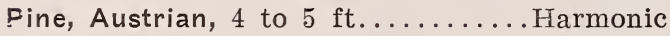

" " $\quad 2$ to $4 \mathrm{ft} \ldots \ldots \ldots \ldots \ldots$. $\quad 2$ to $3 \mathrm{ft} \ldots \ldots \ldots \ldots$.

" 18 to 24 in............. . . . . . . . . . .

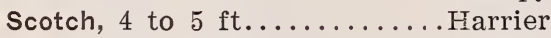

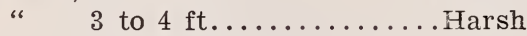

" 2 to $3 \mathrm{ft} . \ldots \ldots \ldots \ldots$ Hartshoril

" 18 to 24 in........... Harvest

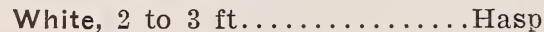

" 18 to 24 in..........Hassock

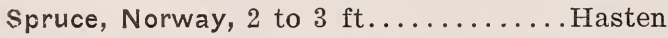

" " 18 to 24 in.......... Hatchel

" " 12 to $18 \mathrm{in........Hatchway}$

VINES.

Code Word. Per 10

Ampelopsis, Veitchii, (Japan Ivy)..... Hatred Quinquefolia, (Va. Creeper).Haulm

Bignonia Radicans, (Trumpet Vine).. Haughty

Clematis, Jackmanii, strong......... Haunch

" Coccinea, strong ......... Hauteur

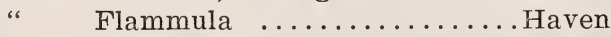

" Paniculata ............. Hawk

Honeysuckle, Hall's Japan...........Hawser

\begin{tabular}{|c|c|}
\hline ( & Evergreen .. \\
\hline & Canadian .............. Hazel \\
\hline
\end{tabular}

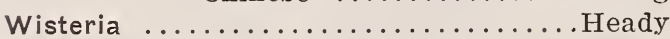

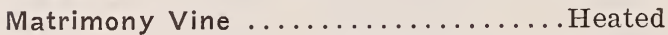

DECIDUOUS SHRUBS.

Code Word.

Altheas, double, 3 to $4 \mathrm{ft} . \ldots \ldots \ldots \ldots$ Hearken

double, 2 to $3 \mathrm{ft} . \ldots \ldots \ldots \ldots$.....................

variegated leaved, 18 to 24 in.. Heart

variegated leaved, 12 to 18 in.. Heath

Almond, double flowering, 18 to 24 in.. Heaven double flowering 2 to $3 \mathrm{ft}$. Heaviness

Berberry, purple leaved, 18 to 24 in..... Heavy

" purple leaved, 12 to 18 in. Hecatomb

"Thunbergii, 18 to 24 in..... Hebraist
$\$ 2.00$

1.50

1.00

2.50

2.00

3.00

2.00

4.00

2.50

2.25

2.00

2.25

2.00

1.75

1.50

2.25

2.00

1.75

1.50

2.50

2.00

2.50

1.75

1.50

Per 100

$\$ 15.00$

12.00

8.00

20.00

18.00

25.00

18.00

35.00

25.00

20.00

18.00

20.00

18.00

15.00

12.50

20.00

18.00

15.00

12.00

20.00

18.00

20.00

15.00

12.00

Per 100

1.50

1.00

1.00

2.00

1.50

1.25

1.20

1.00

1.00

1.00

1.00

1.00

1.00

12.00

8.00

8.00

18.00

15.0)

10.00

10.00

8.00

8.00

8.00

8.00

9.00

8.00

Per 10.

$\$ 1.20$

1.00

1.25

1.00

1.25

1.50

.75

.70

1.00
Per 100. $\$ 10.00$

8.00

12.00

10.00

10.00

12.50

7.00

5.00

8.00 


\begin{tabular}{|c|c|c|}
\hline Code Word. & Per 10. & Per 100. \\
\hline Calycanthus, 18 to 24 in........... Hebrew & 1.00 & 10.00 \\
\hline “ $\quad 12$ to 18 in.............Hectic & .80 & 8.00 \\
\hline Crabs, Bechtel's double flower'g, 2 to & & \\
\hline $\begin{aligned} & 3 \mathrm{ft} \ldots \ldots \ldots \ldots \ldots \ldots \ldots \ldots \ldots \text { Hegira } \\
& \text { " Bechtel's double flower'g, } 18\end{aligned}$ & 1.75 & 15.00 \\
\hline to 24 in .................. Heifer & 1.50 & 12.50 \\
\hline Duetzie, assorted, 2 to $3 \mathrm{ft} \ldots \ldots \ldots$ Heinou: & 1.20 & 10.00 \\
\hline " assorted, 18 to 24 in..........Helm & 1.00 & 8.00 \\
\hline “ assorted, 12 to 18 in........ Helmet & .80 & 6.00 \\
\hline Fringe, Purple, 2 to $3 \mathrm{ft} \ldots \ldots \ldots \ldots \ldots$ Helper & 1.00 & 10.00 \\
\hline " Purple, 18 to 24 in..........Hemper & .80 & 8.00 \\
\hline Honeysuckle, upright, 2 to $3 \mathrm{ft} . . . \ldots$. Henbane & 1.00 & 10.00 \\
\hline " upright, 18 to 24 in...... Hepatic & .80 & 8.00 \\
\hline Hydrangea-Grandiflora, 2 to $3 \mathrm{ft} . .$. . Heptagon & 1.25 & 10.00 \\
\hline " " 18 to 24 in..Heptarchy & 1.00 & 8.00 \\
\hline Japonica, or Japan Quince, 18 to 24 in.Heraldry & 1.00 & 8.00 \\
\hline " $\quad 2$ to $3 \mathrm{ft} . \ldots \ldots \ldots \ldots$ Herbaceous & 1.20 & 10.00 \\
\hline Lilacs, purple, 18 to 24 in...........Herbage & 1.00 & 8.00 \\
\hline " white, 18 to $24 \mathrm{in} . . . \ldots \ldots$. Herbarium & 1.25 & 10.00 \\
\hline " $\quad 2$ to 3 ft purple,...........Herculean & 1.20 & 10.00 \\
\hline Frivet, common, 12 to 18 in........... Herd & .70 & 5.00 \\
\hline " California, 3 to $4 \mathrm{ft} . \ldots \ldots$....Hereditary & .60 & 5.00 \\
\hline " $\quad 2$ to $3 \mathrm{ft} . \ldots \ldots \ldots \ldots$ Heresy & .50 & 3.50 \\
\hline 18 to 24 in........Heretical & .40 & 2.50 \\
\hline 12 to 18 in........Heritage & .30 & 2.00 \\
\hline Prunus Triloba, 2 to $3 \mathrm{ft} \ldots \ldots \ldots \ldots$......... & 1.50 & 10.00 \\
\hline " Pissardi, 2 to $3 \mathrm{ft} . \ldots \ldots \ldots \ldots$ Hermit & 1.50 & 10.00 \\
\hline Syringa, (Mock Orange) 18 to 24 in..... Heroic & 1.00 & 8.00 \\
\hline " (Mock Orange) 12 to 18 in.....Heroism & .80 & 7.00 \\
\hline Spirea, Billardi, 18 to 24 in..........Herring & 1.00 & 8.00 \\
\hline " Van Houttei, 18 to 24 in....... Hesitate & 1.25 & 10.00 \\
\hline Opulifolia Aurea, 12 to 18 in.Heterodox & 1.50 & 12.00 \\
\hline Snowballs, 2 to $3 \mathrm{ft} \ldots \ldots \ldots \ldots \ldots \ldots$ Hew & 1.25 & 10.00 \\
\hline " $\quad 18$ to 24 in.............Hexigon & 1.00 & 8.00 \\
\hline Weigelia, five sorts, 18 to 24 in....... Hexapla & 1.25 & 10.00 \\
\hline " five sorts, 12 to 18 in......Hexapod & 1.00 & 8.00 \\
\hline
\end{tabular}

\section{ROSES.}

Code Word. Per 100. Per 1000.

Hybrid Perpetuals, 2 yrs., No. 1... Hexameter Moss, 2 yrs., and extra 1 yr., strong... Hibernal Climbing, 2 yrs., and extra 1 yr., strong. History Crimson Rambler, 2 yrs., No. 1..... Hibernate Yellow Rambler, 2 yrs., No. 1......Hisbiscus Tree Roses ...................Hideous 


\section{SEEDLINGS AND SCIONS.}

Code Word. Per 100. Per 1000.

Apple Scions, assorted, common sorts...Hiatus assorted, scarce sorts......Hie

$\$ .25$

.25

.30

.30

.30

.25

.25

.25

.25

.25
$\$ 1.00$

1.25

2.00

2.00

2.00

1.50

1.00

1.50

1.00

1.50

California Privet Cuttings............Hillock

For assortment Apple Scions, see last page.

\section{SEEDLINGS.}

Code Word.

Per 1000 .

Pear, French, 3-16 in and up, For. grown...Hilt

$2-16$ to $3-16$ in For. grown. Hindoo

Keiffer, $3-16$ in and up.........Hireling

" " $2-16$ to $3-16$ in........ Hiresute

under $2-16$ in...........Hissing

Cherry, Mahaleb, 5 to $8 \mathrm{~m}-\mathrm{m}$, Fren. gro..Hinge

8.50

" " 3 to $5 \mathrm{~m}-\mathrm{m}$, Fren. gro..Hither

6.00

\section{APPLE SEEDLINGS.}

1 yr., No. 1, 3-16 and all up, straight........

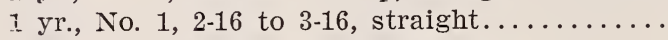

1 yr., No. 1, 3-16 and all up, branched........

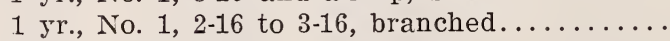

1 yr., under $2-16 \ldots \ldots \ldots \ldots \ldots \ldots \ldots \ldots$

Apple Grafts

WEEPING TREES.

Prices quoted

on

application.

Code Word.

Per 10

Ash, European Mountain, 2 yr., heads... Hives

Birch, Cut-leaved, 6 to $7 \mathrm{ft} . \ldots \ldots \ldots$........

" " 5 to $6 \mathrm{ft} \ldots \ldots \ldots \ldots$ Hobbles

5.00

" " 4 to 5 ft............Hock

4.00

" " 3 to $4 \mathrm{ft} . \ldots \ldots \ldots$........

Elm, Camperdown, No. 1.........Hoggish

Mulberry, Tea's ...............Hogshead

Willow, Kilmarnock, 2 yr., heads........ Hoist

" " 1 yr., heads...... Holiday 
FOREST TREE SEEDLINGS.

All Nursery Grown.

Code Word.

Per 1000

Ash, 1 yr., No. 1,12 to 18 in.........Homicide

$\$ 3.00$

2.50

Box Elder, 1 yr., No. 1, 18 to 24 in.......Hominy

3.00

2.50

“ " " 1 yr., No. 1,12 to 18 in...Homogen

1.75

Plack Locust, 1 yr., 3 to $4 \mathrm{ft} . . . \ldots \ldots$....Honey

5.00

" " 1 yr., 2 to $3 \mathrm{ft} \ldots \ldots \ldots$....Honor

3.00

“ 1 yr., No. 1,18 to 24 in.Honorary

2.50

" " 1 yr., No. 1,12 to 18 in. Hoodlum

2.00

“ 1 yr., No. 1,8 to 12 in..Hoodwink

1.50

3.00

Catalpa, 1 yr., No. 1,18 to 24 in..........Hoof

2.50

1 yr., No. 1,12 to 18 in........Hookah

1.50

Elm, 2 yr., 3 to 4 ft............................

5.00

4.00

3.00

2.50

2.50

1.50

3.00

2.50

3.00

2.50

2.00

1.50

5.00

3.00

2.50

1.50

1.00

1.25

Osage Hedge, 1 yr., No. $1 \ldots \ldots \ldots \ldots$. Hornpipe

1.00

\section{HERBACEOUS PLANTS AND BULBS.}

Code Word. Per 10. Per 100.

Dahlias, mixed colors............Horoscope Dicentra Spectabilis (Bleeding Heart).Horrible

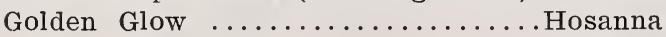
Gladioli, mixed sorts.............Hosiery " named varieties ..........Hospital Hyacinths, assorted colors.......... Hostage

Phlox, mixed colors................. Hostess

Paeonies, mixed ......................tlity

Tiger Lilies ....................... Hotel

Tulips, assorted colors......................

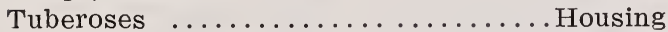
Yucca Filamentosa ...............Hubbub

$\begin{array}{rr}\$ 1.25 & \$ 10.00 \\ 1.25 & 10.00 \\ .75 & 6.00 \\ .50 & 4.00 \\ .75 & 6.00 \\ .75 & 6.00 \\ 1.00 & 8.00 \\ 1.25 & 10.00 \\ .75 & 6.00 \\ .35 & 3.00 \\ .25 & 1.00 \\ 1.25 & \end{array}$

10.00

6.00

4.00

6.00

8.00

10.00

$6.0 \mathrm{C}$

3.00

1.25 


\section{Apple Scions.}

Assorted, common sorts, $\$ 1.00$ per 1000

Assorted, scarce sorts, marked *, \$1.25 per 1000

1500 Alexande:*

7000 A. S. Pearmain

10000 Arkansas Black*

5000 Autumn Strawberry

2500 Big Romanite

3000 Baldwin*

7000 Benoni

4000 Bailey Sweet*

2500 Babbitt

3000 Belmont

4800 Bellflower*

6000 Chenango Strawberry

3600 Chase's Jelly

500 Charlemoff*

10000 Clayton

14000 C. E. White*

9000 Dominie

30000 Duchess*

4800 Early Strawberry

2400 Early Ripe*

2200 Early Pennock

2800 Elk Horn

17000 Early Harvest*

6500 E. G. Russett

21.000 Florence

4200 Fameuse*

14000 Fall Pippin

5000 Flora Bell

36000 G. G. Pippin

86000 Gano

10000 Gideon*

4000 Gravenstein

2800 General Grant*

3800 Golden Sweet

24000 Huntsman

1400 Haas

6000 Hubbardson's

9000 Hybernal*

14000 Hyslop*

7000 Iowa Blush

27000 Ingram

5000 Jeffries

15000 Janet

50000 Jonathan

5000 Kennard's Choice

10000 King

6000 Ky. Red Streak

1200 Loy

7500 Lawver

4700 Lowell

2000 L. S. Pearmain

4000 Lansingburg

8000 Longfield

8000 Minkler

16000 Maiden BIush*

8800 McMahon's W.

2000 Mother

4200 McIntosh Red*

5000 McAfee

6000 Milan

\begin{tabular}{|c|c|}
\hline 20000 & Martha \\
\hline 4000 & Malinda* \\
\hline 6000 & Mann \\
\hline 60000 & Missouri Pippin \\
\hline 40000 & M. B. Twig* \\
\hline $\begin{array}{l}65000 \\
28000\end{array}$ & N. W. Greening \\
\hline $\begin{array}{r}28000 \\
400\end{array}$ & Northern Spy \\
\hline $\begin{array}{r}400 \\
4000\end{array}$ & Porter \\
\hline $\begin{array}{l}4000 \\
8000\end{array}$ & Ozark \\
\hline $\begin{array}{r}8000 \\
800\end{array}$ & $\begin{array}{l}\text { Fatton's Greening* } \\
\text { Peck's Ploant }\end{array}$ \\
\hline 17000 & Pewaukee \\
\hline 9000 & Plum Cider \\
\hline 7000 & Quaker Beauty \\
\hline 10000 & Red Limber Twig \\
\hline 4000 & Rome Beauty* \\
\hline 6000 & Red Beitigheimer \\
\hline 1400 & R. W. Pearmain \\
\hline 11500 & Roman Stem \\
\hline 3200 & Ramsdell's Sweet \\
\hline 4000 & Red Siberian \\
\hline 3600 & R. I. Greening* \\
\hline 7500 & Red Romanite \\
\hline 11600 & Rambo* \\
\hline 10000 & Red Astrachan* \\
\hline 12000 & Red June* \\
\hline 200 & Shannon's Pippin* \\
\hline 9000 & Summer Queen \\
\hline 7000 & Shackleford \\
\hline 6000 & Smith's Cider \\
\hline 5000 & Salome \\
\hline 4400 & Springdale \\
\hline 1400 & Smoke House \\
\hline 17000 & Shockley \\
\hline 4000 & Sutton's Beauty \\
\hline 7000 & Sweet Bough \\
\hline 8000 & Stark \\
\hline 4000 & Seek No Further \\
\hline 6000 & Sops of Wine \\
\hline 24000 & Transcendant \\
\hline 1700 & Talpehockin \\
\hline 2400 & Twenty Oz. Pippin* \\
\hline 8000 & Talman Sweet* \\
\hline 5000 & Utter's Red \\
\hline 3600 & Vandivere Pippin \\
\hline 2600 & Winter Banana* \\
\hline 3000 & Western Beauty \\
\hline 4800 & Wagoner* \\
\hline 9000 & Willow Twig \\
\hline 35000 & Wealthy* \\
\hline 10000 & Whitney \\
\hline 16000 & Walbridge \\
\hline 100000 & Winesap \\
\hline 35000 & York Imperial \\
\hline 40000 & Yellow Transparent \\
\hline 10000 & Yellow Horse* \\
\hline 400 & Yellow Siberian \\
\hline 800 & Wismer's Desert* \\
\hline 1000 & W. W. Pearmain* \\
\hline 26000 & Wolf River* \\
\hline
\end{tabular}

These Scions are from a young scion orchard and are of excellent quality.

\section{F. H. Stannard \& Co., OTTAWA, KANSAS.}

